ISSN 2306-1561

Automation and Control in Technical Systems (ACTS)

2014, No 1.2(9), pp. 149-157.

DOI: $10.12731 / 2306-1561-2014-1-28$

\title{
Control algorithms connected dosing multicomponent ceramic mixtures
}

\section{Ilukhin Andrey Vladimirovich}

Russian Federation, Doctor of Technical Sciences, Professor, Head of Department of «Automation Industrial Process».

State Technical University - MADI, 125319, Russian Federation, Moscow, Leningradsky prospekt, 64. Tel.: +7 (499) 151-64-12. http://www.madi.ru

\section{aviluhin@mail.ru}

\section{Kolbasin Alexander Markovich}

Russian Federation, Ph.D., Associate Professor, Department of «Automation Industrial Process».

State Technical University - MADI, 125319, Russian Federation, Moscow, Leningradsky prospekt, 64. Tel.: +7 (499) 151-64-12. http://www.madi.ru

\section{alex123456789.a@yandex.ru}

\section{Abdulkhanova Marina Yurievna}

Russian Federation, Ph.D., Associate Professor, Department of «Automation Industrial Process».

State Technical University - MADI, 125319, Russian Federation, Moscow, Leningradsky prospekt, 64. Tel.: +7 (499) 151-64-12. http://www.madi.ru

\section{Dinh An Ninh}

People's Republic of China, Postgraduate Student, Department of «Automated Control Systems».

State Technical University - MADI, 125319, Russian Federation, Moscow, Leningradsky prospekt, 64. Tel.: +7 (499) 151-64-12. http://www.madi.ru

\begin{abstract}
The article describes the processes connected Batching ceramic mixes. This allows you to combine separate metering devices into a single system of preparation of doses that minimize errors in the content of its components.
\end{abstract}

Keywords: dosing, control, processes and manufacturing industries, extreme control system (ECS), construction mixtures. 
ISSN 2306-1561

Автоматизация и управление в технических системах (АУТС) 2014. - №1.2(9). - C. 149-157.

DOI: $10.12731 / 2306-1561-2014-1-28$

УДК 681.3

\section{Алгоритмы управления связным многокомпонентным дозированием керамических смесей}

\section{Илюхин Андрей Владимирович}

Российская Федерация, доктор технических наук, профессор, заведующий кафедрой «Автоматизация производственных процессов».

ФГБОУ ВПО «Московский автомобильно-дорожный государственный технический университет (МАДИ)», 125319, Российская Федерация, г. Москва, Ленинградский проспект, д.64, Тел.: +7 (499) 151-64-12, http://www.madi.ru

\section{aviluhin@mail.ru}

\section{Колбасин Александр Маркович}

Российская Федерация, кандидат технических наук, доцент кафедры «Автоматизация производственных процессов».

ФГБОУ ВПО «Московский автомобильно-дорожный государственный технический университет (МАДИ)», 125319, Российская Федерация, г. Москва, Ленинградский проспект, д.64, Тел.: +7 (499) 151-64-12, http://www.madi.ru

\section{alex123456789.a@yandex.ru}

\section{Абдулханова Марина Юрьевна}

Российская Федерация, кандидат технических наук, доцент кафедры «Автоматизация производственных процессов».

ФГБОУ ВПО «Московский автомобильно-дорожный государственный технический университет (МАДИ)», 125319, Российская Федерация, г. Москва, Ленинградский проспект, д.64, Тел.: +7 (499) 151-64-12, http://www.madi.ru

\section{Динь Ан Нинь}

Китайская Народная Республика, аспирант кафедры «Автоматизация производственных процессов».

ФГБОУ ВПО «Московский автомобильно-дорожный государственный технический университет (МАДИ)», 125319, Российская Федерация, г. Москва, Ленинградский проспект, д.64, Тел.: +7 (499) 151-64-12, http://www.madi.ru 
Аннотация. В статье рассмотрены технологические процессы связного дозирования компонентов керамических смесей. Это позволяет объединить отдельные дозирующие устройства в единую систему приготовления доз, обеспечивающую минимизацию погрешностей содержания в ней компонентов.

Ключевые слова: дозирование, управление, технологические процессы и производства, промышленные предприятия, системы экстремального регулирования (СЭР), строительные смеси.

\section{1. Введение}

Многокомпонентное дозирование, является неотъемлемой частью технологических процессов приготовления смесей самого различного назначения, в том числе и керамических [1 - 15]. Известно несколько структурных схем реализации такого дозирования, которые можно объединить в две группы:

- не связное дозирование, при котором каждый компонент смеси дозируется в соответствии с заданной программой и без учета результатов дозирования остальных компонентов;

- связное дозирование, при котором перед началом дозирования очередного компонента анализируется результат дозирования предыдущих компонентов смеси и на основе принятого критерия оптимизации процесса в программу дозирования очередного компонента вносятся соответствующие изменения.

Не связное дозирование широко применяется на практике, а связное изучено пока недостаточно.

\section{2. Основы принципов управления процессом дозирования}

Рассмотрим структурные схемы управления процессом дозирования, используемых в практике производства многокомпонентных смесей.

При несвязном «традиционном» дозировании, результат отмеривания дозы отдельного компонента смеси не влияет на процесс дозирования остальных компонентов [1 - 6]. Это позволяет все компоненты смеси дозировать одновременно, в один этап.

Двухэтапное дозирование позволяет на первом этапе дозирования компонентов обеспечить загрузку весовой емкости в «грубом» режиме взвешивания, при котором взвешивается основная масса материала. На втором этапе дозирования, в режиме «досыпки» питатель переводится на пониженную производительность, обеспечивая точное получение заданной дозы. Такой принцип дозирования уменьшает влияние динамического воздействия столба материала на подверженную колебаниям весоизмерительную систему дозатора, повышая тем самым качество дозирования. Требуемая точность в этом случае достигается за счет снижения производительности оборудования. Поэтому «традиционная» схема циклического многокомпонентного 
дозирования имеет преимущество перед двухэтапной схемой по быстродействию и оперативности.

При не связном многокомпонентном весовом дозировании задается величина результирующей массы смеси $V_{p o}$. Долевое содержание каждого компонента в массе смеси $V_{p o}$, дает рецепт смеси. Значения уставок задатчиков дозаторов, определяются из соотношения:

$$
U_{i}=\gamma_{i} \cdot V_{p o} ; i=1,2,3
$$

Результирующая масса смеси $V_{p o}$, представляет собой сумму заданных доз компонентов $X_{i 0} ; \gamma_{i}$ - коэффициент долевого содержания в заданной массе смеси, массы і -го компонента.

Коэффициент $\gamma_{i}$ равен отношению заданной дозы компонента $X_{i 0}$ к результирующей массе смеси $V_{p o}$ :

$$
\gamma_{i}=X_{i 0} / V_{p o},(i=1,4)
$$

Одновременное не связное дозирование компонентов начинается после назначения уставок задатчиков дозаторов $U_{i}$.

Алгоритм управления не связным дозированием, наряду с операциями вычисления уставок задатчиков дозаторов компонентов, включает в себя ряд других операций: управление затворами питателей, подключение металлоуловителя, включение сводообрушителя, при «зависании» материала в расходном бункере и другие.

После окончания цикла дозирования, система управления приходит в исходное состояние и при повторении цикла система управления вновь повторяет заданную программу дозирования.

При связном дозировании все или часть компонентов смеси $n_{j},\left(n_{j}<n\right)$ дозируются в интервалы, разделенные по времени. При завершении цикла дозирования, например, компонента $X_{i}$, можно определить его отдозированную фактическую массу $X_{i}\left(U_{i}\right)$ при заданной уставке $U_{i}$. Измерение отдозированной массы компонента осуществляется в установившемся режиме и оценивается статической погрешностью дозирования:

$$
\Delta X_{i}=X_{i}\left(U_{i}\right)-U_{i} ; i=1, \ldots n,
$$

Можно использовать значение оценки массы от дозированного компонента для коррекции доз компонентов, дозируемых на следующих этапах. Такая коррекция позволяет добиться заданного качества многокомпонентной смеси, которая зависит от точности соблюдения заданной рецептуры. 


\section{3. Влияние корректирующих связей на точность дозирования}

Введение корректирующих связей между дозаторами, обеспечивает связное дозирование компонентов смеси в установленной очередности.

При наличии корректирующих связей уставка задатчика дозатора компонента $X_{i}$, дозируемого на очередном этапе, определяется функциональной связью общего вида:

$$
U_{i}=F\left[X_{1}\left(U_{1}\right), X_{2}\left(U_{2}\right), \ldots, X_{i-1}\left(U_{i-1}\right)\right] ; \quad i=1, n
$$

где $X_{i}\left(U_{i}\right)$ - измеренные массы отдозированных компонентов $X_{1}, X_{2}, \ldots, X_{i-1}$. Конкретный вид функциональной связи (2) зависит от принятого способа коррекции, связывающего массы компонентов смеси.

Если выбрать схему связного циклического многокомпонентного дозирования с «ведущим» дозатором, то условие, связывающее корректируемые массы компонентов, будет:

$$
\beta_{i}=X_{i 0} / X_{j 0} ; i=1, n ; j=1, n ; i \neq j
$$

где $X_{i 0}$ - заданная масса компонента «ведущего» дозатора; $X_{j 0}$ - заданные массы компонентов смеси «ведомых» дозаторов.

Если отдозированная на первом этапе масса компонента $X_{i 0}$ «ведущего» дозатора не равна заданной, то для соблюдения условия (3), т.е. правило, по которому будут корректироваться дозы компонентов «ведомых» дозаторов, будет:

$$
U_{i+1}=X_{1}\left(U_{1}\right) / \beta_{i} ; \mathrm{i}=1, \mathrm{n}-1,
$$

где $X_{l}\left(U_{l}\right)$ - отдозированная масса компонента «ведущего» дозатора; $U_{l}=X_{1}$ уставка задатчика «ведущего» дозатора; $U_{i+1}, \quad(i=1, n-1)$ - скорректированные уставки задатчиков «ведомых» дозаторов.

Если выбрать условие связи между массами компонентов смеси в виде:

$\beta_{i}=X_{(i-1) 0} / X i O ; i=2, n$,

то после дозирования компонента $\mathrm{Xi}-1$ уставка задатчика дозатора компонента $X_{i}$, дозируемого на $j+1$ этапе, определится из соотношения:

$$
U_{j+1}=\gamma_{j+1} \cdot X_{j}\left(U_{j}\right) / \gamma_{j} ; \mathrm{j}=1, \mathrm{n}-1,
$$

где $X_{j}\left(U_{j}\right)$ - отдозированная на $j$ - ом этапе масса компонента $X_{i l}$; $\gamma_{j}=X_{i 0} / V_{p o},(\mathrm{i}=\mathrm{j}=1, \mathrm{n})$ - коэффициенты долевого содержания компонентов смеси; $U_{j+1}$ - уставка задатчика дозатора компонента $X_{i}$, дозируемого на $j+1$-м этапе.

Так, если величина заданной результирующей массы компонентов смеси - Vpo, то в соответствие с заданной уставкой:

$$
U_{1}=\gamma_{1} \cdot V_{p o}=X_{1}
$$

производится дозирование первого компонента первым дозатором. В соответствие с величиной измеренной массой $X_{l}\left(U_{l}\right)$ первого от дозированного 
компонента $X_{1}$ корректируется уставка дозы следующего компонента $X_{2}$, дозируемого на втором этапе:

$$
U_{2}=X_{1}\left(U_{1}\right) \cdot \gamma_{2} / \gamma_{1} \text {. }
$$

Аналогично последовательно корректируются уставки дозаторов остальных компонентов смеси. Так, уставка задатчика дозатора компонента Xn , дозируемого на $\mathrm{n}$ - ом этапе, будет равна:

$$
U_{n}=X_{n-1}\left(U_{n-1}\right) \cdot \gamma_{n} / \gamma_{n-1} \text {. }
$$

По окончании цикла дозирования результирующая масса смеси будет равна сумме отдозированных масс компонентов - $X_{1}\left(U_{1}\right), X_{2}\left(U_{2}\right), \ldots, X_{n}\left(U_{n}\right)$.

Приращение результирующей массы смеси $\Delta V_{p}$ по окончании цикла дозирования будет зависеть как от условия, связывающего массы $i$-го и $j$ - го компонентов, так и от очередности их дозирования.

Кроме рассмотренных выше условий связного управления дозами компонентов, может быть использовано условие управления дозами компонентов по критерию «средней скользящей массы» и условие, когда уставка задатчика дозатора $i$ - го компонента, дозируемого на очередном этапе, корректируется по значениям погрешностей дозирования предыдущих компонентов на основе минимаксного критерия.

В первом варианте, перед очередным $j$-ым этапом дозирования, уставка этого дозатора корректируется по величине массы $\overline{V_{j}}$, определяемой как среднее значение мacc $V_{l}, V_{2}, \ldots, V_{j-1}$ :

$$
U_{j}=\gamma_{j} \cdot \overline{V_{j}}=\gamma_{j} \cdot \sum_{i=1}^{j-1} \frac{V_{i}}{j-1} ; \mathrm{i}=1, \mathrm{j}-1,
$$

где $V_{i}=X_{i}\left(U_{i}\right) / \gamma_{i}$ - масса смеси, обеспечивающая нулевую погрешность дозирования $i$-го компонента; $\gamma_{i}$ - коэффициент долевого содержания $i$-го компонента в заданной массе $V_{p o}$; $U_{j}$ - скорректированная уставка задатчика дозатора $j$ - го компонента, дозируемого на очередном $j$ - м этапе.

Во втором варианте доза компонента перед очередным $j$-ым этапом дозирования корректируется по значению величины $\bar{V}_{j}$, представляющей собой среднее значение максимума и минимума масс $\mathrm{Vi},(\mathrm{i}=1, \mathrm{j}-1)$ :

$$
U_{i}=\frac{\gamma_{i}\left[\max \left\{V_{1}, V_{2}, \ldots, V_{j-1}\right\}+\min \left\{V_{1}, V_{2}, \ldots, V_{j-1}\right\}\right]}{2} .
$$

Если при не связном многокомпонентном дозировании повышение точности дозирования достигается за счет улучшения метрологических характеристик весодозирующих элементов дозатора, то при связном дозировании эта задача решается поддержанием в процессе дозирования заданных массовых соотношений между компонентов. 


\section{4. Особенности связного дозирования}

Поэтапная коррекция в процессе дозирования, увеличивает время цикла дозирования. Это снижает производительность смесительной установки. Вместе с тем при параллельной работе дозаторов на один смеситель, времена цикла дозирования и перемешивания смеси находятся в соотношении $1 / 5$, что определяет резерв времени вынужденного «простоя» дозаторов, который можно использовать для организации работы системы в режиме связного дозирования.

При связном дозировании увеличивается коэффициент вариации результирующей массы $V_{p}$. Это связано с тем, что погрешность дозирования $i$ - го компонента компенсируется изменением дозы компонента, дозируемого в установленной очередности после $i$ - го.

Управление дозированием компонентов смеси реализуется при выполнении условий:

$$
X_{i}^{H} \leq X_{i}^{\Phi} \leq X_{i}^{B}
$$

или

$$
\left|\Delta X_{i}\right| \leq \Delta X_{i}^{\text {don }}
$$

где $X_{i}^{\Phi}$ - отдозированная масса $i$-го компонента; $X_{i}^{H}, X_{i}^{B}$ - соответственно допустимые нижняя и верхняя границы изменения отдозированной массы $i$ - го компонента; $\Delta X_{i}=X_{i}^{\Phi}-X_{i 0}, \Delta X_{\mathrm{i}}^{\text {доп. }}$ - полученная при дозировании допустимая абсолютная ошибка і - го компонента.

Для относительной погрешности дозирования $i$-го компонента:

$$
\left|\frac{\Delta X_{i}}{\Delta X_{i 0}}\right| \leq \delta_{i}^{\text {don }}
$$

где $\delta_{\mathrm{i}}^{\text {доп. }}-$ допустимая относительная погрешность дозирования $\mathrm{i}$-го компонента.

Для получения заданной рецептуры смеси, при многокомпонентном циклическом дозировании можно воспользоваться соотношением:

$$
\gamma_{i}^{H} \leq \gamma_{i}^{\Phi} \leq \gamma_{i}^{B} ; \mathrm{i}=1, \mathrm{n},
$$

где:

$$
\gamma_{i}^{H}=\frac{X_{i}^{H}}{V_{p}^{H}} ; \quad \gamma_{i}^{B}=\frac{X_{i}^{B}}{V_{p}^{B}} ; \quad \gamma_{\mathrm{i}}^{\Phi}=\frac{X_{i}^{\Phi}}{\sum_{i=1}^{n} X_{i}^{\Phi}}=\frac{X_{i}^{\Phi}}{V_{p}} .
$$

Величина абсолютной погрешности дозирования i - го компонента бетонной смеси с учетом ее результирующей массы, будет:

$$
\Delta U_{i}=X_{i}\left(U_{i}\right)-\gamma_{i} \cdot V_{p} ; \quad \mathrm{i}=1, \mathrm{n}
$$


где $X_{i}\left(U_{i}\right)=U_{i} \pm \Delta X_{i}$ - отдозированная масса $i$-го компонента; $U_{i}$ - уставка задатчика дозатора $i$-го компонента; $\Delta X_{i}$ - абсолютная погрешность, дозирования $i$-го компонента; $V_{p}$-результирующая масса смеси в $k$-ом цикле дозирования $(k=1, N)$ :

$$
V_{p}=\sum_{i=1}^{n} X_{i}\left(U_{i}\right)=\sum_{i=1}^{n} U_{i}+\sum_{i=1}^{n} \Delta X_{i} ; \quad \mathrm{i}=1, \mathrm{n} .
$$

Величина $\gamma_{i} \cdot V_{p}=X_{i 0}^{*}$ - расчетная масса $i$-го компонента, обеспечивающая нулевую погрешность дозирования $i$-го компонента - $\Delta \gamma_{i}=0$ :

$$
\Delta \gamma_{i}=\frac{X_{i 0}^{*}}{V_{p}}-\frac{X_{i 0}}{V_{p 0}}=0 ; \mathrm{i}=1, \mathrm{n} .
$$

Разделив обе части (16) на заданный коэффициент долевого содержания массы i го компонента в заданной массе смеси $\gamma_{i},(i=1, n)$, получим:

$$
\Delta V_{i}=V_{i}-V_{p} ; \quad \mathrm{i}=1, \mathrm{n},
$$

где $V_{i}$ - текущая масса смеси, при которой погрешность дозирования $\Delta \gamma_{i}$, полученная на каждом этапе связного многокомпонентного дискретного дозирования, равна нулю.

\section{5. Заключение}

Связное дозирование компонентов строительных смесей позволяет, объединив отдельные дозаторы циклического действия в единую систему с взаимозависимыми процессами дозирования, использовать механизм взаимокомпенсации ошибок дозирования на каждом этапе набора доз отдельных компонентов, приближая процентные содержания компонентов в результирующей массе к оптимуму.

\section{Список информационных источников}

[1] Марсов В.И., Тихонов А.Ф., Бокарев Е.И. Дозирующие системы непрерывнлого действия нового поколения // Механизация строительства. - 2012. - №3. - С. 27 30.

[2] Васильев Ю.Э., Марсов В.И., Гиляровский Т.В. Оптимизация динамических процессов комбинированной системы дозирования // Механизация строительства. - 2012. - №8. - С. 40-43.

[3] Марсов В.И., Суэтина Т.А., Колбасин А.М., Тан Цзиюя. Алгоритм управления оптимальной системы пневмодозирования сыпучих материалов // Автоматизация и управление в технических системах. - 2013. - № 1(3). - С. 39-44.

[4] Колбасин А.М., Тан Цзиюя, Марсова Е.В., Шухин В.В. Система экстремального регулирования потока материала в пневмосистеме // Автоматизация и управление в технических системах. - 2013. - № 1(3). - С. 44-48.

[5] Марсов В.И., Колбасин А.М., Тан Цзиюя, Шухин В.В. Система оптимального регулирования процессом пневмотранспортирования // Автоматизация и управление в технических системах. - 2013. - № 1(3). - С. 48-52. 
[6] Марсов В.И., Колбасин А.М., Сарычев И.Ю., Курилин А.В. Методы улучшения качественных характеристик системы экстремального регулирования // Автоматизация и управление в технических системах. - 2013. - № 3(5). - С. 3-6.

[7] Остроух А.В. Автоматизация и моделирование работы предприятий по строительству промышленных объектов: дис. ... д-ра техн. наук: 05.13.06: защищена 07.04.09: утв. 19.06.09. - М., 2009. - 357 с.

[8] Остроух А.В. Автоматизация и моделирование работы предприятий по строительству промышленных объектов: автореф. дис. ... д-ра техн. наук: 05.13.06. - М., 2009. - 43 c.

[9] Остроух А.В. Исследование начального периода моделирования на точность среднеинтегральной оценки имитационных моделей / А.В. Остроух, А.А. Солнцев, Н.В. Солдатов, К.А. Новицкий, П.С. Якунин // Вестник МАДИ. - 2010. Вып. 2(21). - С. 61-65.

[10] Остроух А.В. Автоматизация технологической линии формирования, перемещения, сушки и обжига на предприятиях кирпично-черепичного производства / А.В. Остроух, Р.Р. Чаудхари, Н.Е. Суркова // Промышленные АСУ и контроллеры. - 2012. - №7. - С. 1-5.

[11] Остроух А.В., Тянь Юань. Современные методы и подходы к построению систем управления производственно-технологической деятельностью промышленных предприятий // Автоматизация и управление в технических системах. - 2013. - № 1(3). - C. 29-31.

[12] Остроух А.В. Интеграция компонентов системы мониторинга / А.В. Остроух, Юань Тянь // Молодой ученый. - Чита: ООО «Издательство Молодой ученый», 2013. - №10. - С. 182-185.

[13] Чаудхари P.Р., Остроух А.В., Суркова Н.Е. Автоматизация технологических процессов производства керамического кирпича // Автоматизация и управление в технических системах. - 2013. - № 1(3). - С. 31-35.

[14] Остроух А.В., Чаудхари Р.Р. Автоматизированная система управления технологическим процессом производства керамического кирпича // Автоматизация и управление в технических системах. - 2013. - № 2(4). - С. 82-85.

[15] Tian Yuan, Ostroukh A.V. Monitoring environment condition of Chinese industrial enterprises // EUROPEAN JOURNAL OF NATURAL HISTORY. - 2013. - № 5 - C. 34-35. 